\title{
Myeloid Sarcoma Presenting with Quadriparesis: A Difficult Journey through COVID Times-Lessons Learned
}

\author{
Sayantan Chakraborty ${ }^{1}$, Manali Chandra ${ }^{2}$, Joydeep Ghosh ${ }^{3}$, Anupam Sarkar ${ }^{4}$
}

\begin{abstract}
A 16-year-old girl presented with acute-onset quadriparesis with urinary incontinence. Examination revealed an upper motor neuron (UMN) pattern of weakness in all four limbs, accompanied by a UMN type of bladder involvement in a shock stage. Two significant cervical lymph nodes were noted in the right submandibular region. MRI of the cervical spine revealed an extradural mass, compressing the cord at the C2 to C4 region. Excisional biopsy of the cervical lymph nodes revealed an extramedullary myeloid sarcoma, and immunohistochemistry showed CD117 and myeloperoxidase positivity.

Keywords: Compressive, Myeloid, Myelopathy, Sarcoma.

Bengal Physician Journal (2021): 10.5005/jp-journals-10070-7041
\end{abstract}

\section{INTRODUCTION}

Quadriparesis is defined as partial weakness of all four limbs. It commonly occurs after a high cervical cord injury. The injury can be divided on the basis of onset of illness. Acute-onset quadriparesis occurring within 1-2 days can have multiple causes, like trauma, vascular, inflammatory, etc. Slowly developing quadriparesis over weeks to months also has various etiologies, like infection, tumor, degeneration, and others. This is a report of a case where a myeloid tumor causes acute-onset quadriparesis.

\section{Case Description}

A 16-year-old Indian girl, student of class 9 and resident of Habra, West Bengal, presented with acute-onset weakness in all four limbs for the last 12 hours and retention of urine for the same duration. She also had shortness of breath. There was a history of radicular neck pain for the last 10 days which was occasional, mild to moderate in grade. No history of any preceding fever, upper respiratory tract infections, or diarrhea-like illnesses. No history of trauma or heavy weight-bearing. No history of headache, vomiting, or altered sensorium.

On examination, she was alert, conscious, and oriented. GCS was 15/15. Pulse $88 /$ minute and regular; BP 100/70 mmHg; RR 24/ minute, thoracic type of breathing, and single-breath count was $16 /$ minute. No evidence of papilledema on ophthalmoscopy was found. General examination did not reveal any abnormalities except two significant, firm, mobile cervical lymph nodes palpable in the right submandibular region. On neurological examination, higher function and speech were normal, with no cervical or spinal tenderness and no cranial nerve involvement. Spasticity was present in all four limbs; power was $2 / 5$ in shoulder abductors, elbow flexors and extensors, wrist flexors and extensors, knee flexors and extensors, and ankle plantar flexors and dorsiflexors. All upper and lower limb jerks were exaggerated, no clonus was present, and plantars were bilaterally extensors. All types of sensations were diminished symmetrically in all four limbs. There was no definite upper level of sensory loss as per the patient and even after a thorough front-and-back chest examination. The bladder was palpable per abdomen and there was evidence of \begin{tabular}{l}
\hline${ }^{1-4}$ Department of General Medicine, Medical College and Hospital, \\
Kolkata, West Bengal, India
\end{tabular} Corresponding Author: Sayantan Chakraborty, Department of General Medicine, Medical College and Hospital, Kolkata, West Bengal, India, e-mail:sc9163@gmail.com

How to cite this article: Chakraborty S, Chandra M, Ghosh J, et al. Myeloid Sarcoma Presenting with Quadriparesis: A Difficult Journey through COVID Times-Lessons Learned. Bengal Physician Journal 2021;8(1):15-16.

Source of support: Nil

Conflict of interest: None

overflow incontinence (postvoid residual urine came out to be $800 \mathrm{~mL}$ ). She was catheterized then.

Routine blood examination did not reveal any abnormalities. MRI of the cervical spine showed an extradural mass, encasing and compressing the cervical cord at the level of $\mathrm{C} 2$ to $\mathrm{C} 4$ region. A cervical lymph node excision was done for histopathological examination which revealed an extramedullary myeloid sarcoma with CD117 and myeloperoxidase (MPO) positivity in immunohistochemistry. MRI of the lumbosacral spine revealed no abnormalities. So, the bladder was likely in a shock stage at that time (Figs $1 \mathrm{~A}$ to $\mathrm{C}$ ).

\section{Clinical Course in the Hospital}

After receiving the lymph node excisional biopsy with immunohistochemistry report, hematology and neurosurgery opinions were taken for further management. No neurosurgical intervention was done by the department of neurosurgery and, according to them, she had systemic disease with both medullary and extramedullary involvement and chemotherapy was the first treatment option. They also opined that she might require surgical intervention if the weakness was not improved by chemotherapy alone. As per hematology opinion, she was planned for a bone marrow aspiration and biopsy to look for bone marrow involvement and also to look for a chromosomal abnormality. Bone marrow biopsy revealed the presence of $5 \%$ myeloid blast cell in the marrow. 

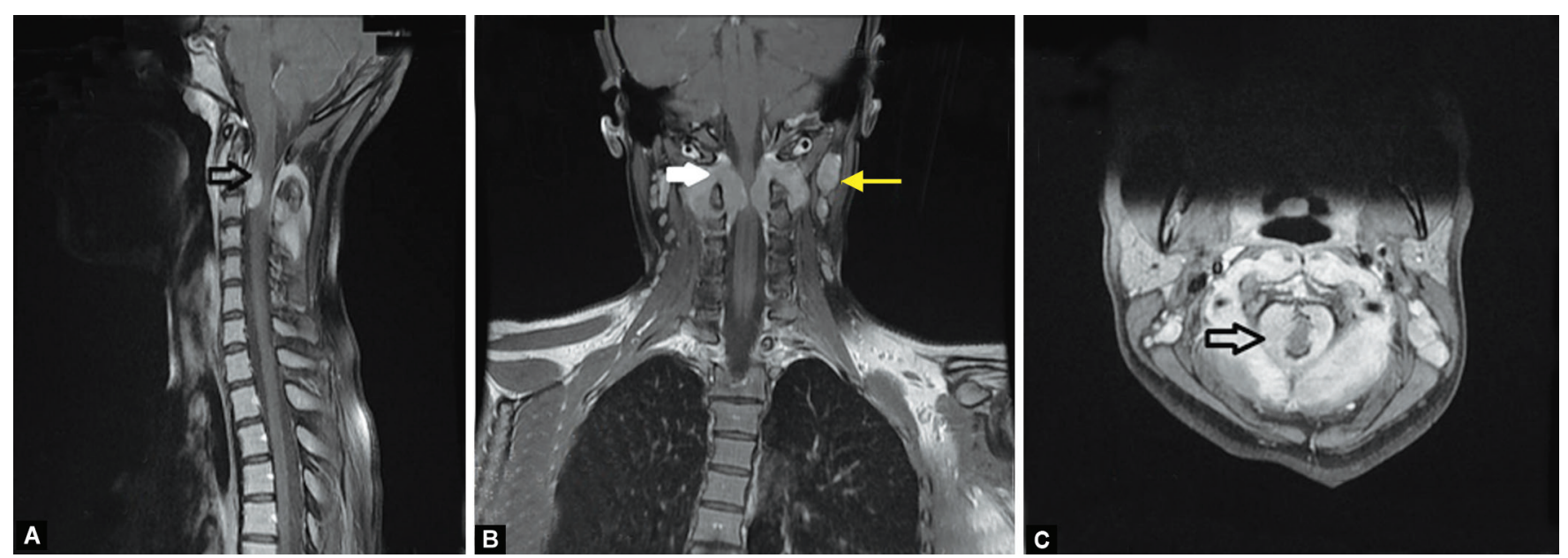

Figs 1 A to C: (A) Sagittal cut section shows enhancing soft tissue mass, encroaching and compressing the spinal cord (black arrow); (B) Coronal cut section shows an enhancing mass, compressing the spinal cord (white arrow) and cervical lymphadenopathy (yellow arrow); (C) Axial cut shows an enhancing mass, encasing and compressing the spinal cord (black arrow)

Subsequent cytogenetic analysis revealed chromosome (8:21) translocation. As per their opinion, the patient was transferred to their department for initiation of chemotherapy. She received her first cycle of chemotherapy ( 3 days of daunorubicin plus 7 days of cytarabine regimen). Currently, the patient is under hematology follow-up. After the first cycle of chemotherapy, the power of her knee flexors and extensors and elbow flexors and extensors has improved to $3 / 5$. She is scheduled to come for follow-up with us next week.

\section{Discussion}

Hematological malignancies can be manifested as extramedullary soft tissue masses in relatively rare cases. One of the rarest manifestations is myeloid sarcoma (MS). It develops as a part of acute myeloid leukemia, myeloproliferative neoplasm (MPN) or myelodysplastic syndrome or at relapse, especially following allogeneic stem cell transplantation. ${ }^{1}$ European Association for Haematopathology has divided this extramedullary myeloid neoplasm into four groups: (1) isolated MS, (2) MS with concurrent $A M L$, (3) extramedullary relapse of AML, and (4) blast transformation of MPN or CML. ${ }^{2}$ It commonly involves lymph nodes, soft tissues, bones, testes, peritoneum, and rarely the central nervous system. ${ }^{1}$ Meena et al. reported a similar case in a child presenting with a CNS mass in acute myeloid leukemia. ${ }^{3}$ Diagnosis is challenging and relies on radiology, histology, immunophenotyping, and molecular analysis which are also essential for risk stratification and treatment planning. ${ }^{4}$ Immunohistochemistry shows CD68-KP1 as the most commonly expressed marker, then MPO, CD117, CD34, and others. ${ }^{1}$ Systemic chemotherapy using AML-like regimens should be commenced early, even in a nonleukemic disease. Surgery and/ or radiotherapy may be indicated for tumors not responding or causing an obstruction. ${ }^{4}$

A direct fine needle aspiration cytology from the mass was not done, but diagnosis and chemotherapy were started on the basis of immunohistochemistry of the lymph node biopsy coupled with the bone marrow cytogenetics showing 8:21 translocation which accompanies MS. However, the patient has started to show improvement in muscle power after the first cycle and is currently undergoing physiotherapy. It is also very important to ensure functionality and better quality of life in this young patient, and we are trying to cope with the socioeconomic, infrastructural, and situational (pandemic) impediments presented to us and help her.

\section{ACKnOWledgments}

Consent has been taken from the patient for publication of the case. We would like to express our gratitude to the patient for her full cooperation.

We also would like to express our thanks to the department of hematology and neurosurgery.

\section{Contributory Statement}

SC prepared and edited the manuscript and was directly involved in patient management. MC and JD helped with clinical correlation and diagnosis and supervised the treatment. AS supervised the entire workup, management, and manuscript preparation, revision, and editing. All authors were in agreement to be accountable for all aspects of the work in ensuring that questions related to the accuracy or integrity of any part of the work were appropriately investigated and resolved.

\section{References}

1. Magdy M, Abdel Karim N, Eldessouki l, et al. Myeloid sarcoma. Oncol Res Treat 2019;42(4):224-229. DOI: 10.1159/000497210.

2. Wilson CS, Medeiros LJ. Extramedullary manifestations of myeloid neoplasms. Am J Clin Pathol 2015;144(2):219-239. DOI: 10.1309/ AJCPO58YWIBUBESX.

3. Meena JP, Yadav M, Gupta AK, et al. Acute myeloid leukemia presenting as a central nervous system mass in a child: a case report. J Pediatr Neurosci 2018;13(1):84-87. DOI: 10.4103/JPN. JPN_152_17.

4. Almond LM, Charalampakis M, Ford SJ, et al. Myeloid sarcoma: presentation, diagnosis, and treatment. Clin Lymphoma Myeloma Leuk 2017;17(5):263-267. DOI: 10.1016/j.clml.2017.02.027. 